\title{
El lugar de la diferencia en Chile: circo y transformismo. El caso del Circo Timoteo
}

\author{
Hugo Capellà Miternique \\ Universidad de Concepción, Chile. Departamento de Geografía \\ hcapella@udec.cl
}

Recepción: octubre de 2011

Aceptación: mayo de 2012

\section{Resumen}

El modelo social chileno ha cedido tradicionalmente poco lugar formal a las diferencias. No obstante, encontramos, en ciertos espacios marginales, instancias en las cuales han podido prosperar curiosos rincones de libertad y de tolerancia. El objetivo del presente artículo es comprender cómo el rol marginal del circo fue clave para la popularización del transformismo.

El caso del Circo Timoteo representa el resultado de un sincretismo cultural, de herencia europea y mesoamericana, en torno al humor y a los roles sexuales, que nos adentra a una aproximación mucho más compleja que una simple realidad social formal unívoca. La figura del género cruzado adopta, en el circo chileno, un espacio propio respecto a la ambigüedad de los roles sexuales y sociales desde un sincretismo cultural criollo. La diferencia adquiere su espacio desde el margen de la ambigüedad formal.

En primer lugar, nos adentraremos en entender teóricamente el ambiguo rol del transformista en el circo criollo chileno, desde su estigmatización hasta su empoderamiento desde la burla. En segundo lugar, nos centraremos en el análisis histórico y etnográfico del caso del Circo Timoteo, en la ambivalencia de ser considerado tanto como elemento genuino del circo criollo chileno, como enfermedad de trascendencia social. En tercer y último lugar, nos detendremos en el análisis lingüístico de los discursos de los transformistas del Circo Timoteo, para demostrar su ambivalencia compleja, como espacio de la diferencia a la criolla.

Palabras clave: circo; diferencia; lugar; transformismo; criollo.

Resum. El lloc de la diferència a Xile: circ i transformisme. El cas del Circo Timoteo

El model social xilè ha cedit tradicionalment poc lloc formal per a les diferències. Amb tot, trobem, en alguns espais marginals, institucions en les quals s'han pogut establir curiosos racons de llibertat i tolerància. L'objectiu del present article és entendre com el rol marginal del circ ha estat clau per a la popularització del transformisme. 
El cas del Circo Timoteo representa el resultat d'un sincretisme cultural, hereu de la tradició europea i mesoamericana, al voltant de l'humor i dels rols sexuals, que ens endinsa en una aproximació molt més complexa que la simple realitat social formal unívoca. La figura del transformisme adopta, al circ xilè, un espai propi respecte a l'ambigüitat dels rols sexuals i socials, des d'un sincretisme cultural propi. La diferència obté el seu lloc des del marge de l'ambigüitat formal.

En primer lloc, ens endinsarem a entendre teòricament el rol ambigu del transformista dins del circ xilè, des de l'estigmatització que pateix, fins a l'apoderament que realitza des de la burla. En segon lloc, ens centrarem en l'anàlisi històrica i etnogràfica del cas del Circo Timoteo, des de l'ambivalència entre un gènere propi dins del circ xilè i com a malaltia de transcendència social. En tercer i darrer lloc, ens aturarem a l'anàlisi lingüística dels discursos dels transformistes del Circo Timoteo, per demostrar-ne l'ambivalència complexa, com un espai de la diferència a la xilena.

Paraules clau: circ; diferència; lloc; transformisme; mestissatge.

Résumé. La place de la différence au Chili: cirque et transformisme. Le cas du Circo Timoteo

Le modèle social chilien a laissé traditionnellement peu de place formelle pour les différences. Malgré tout, nous trouvons dans certains espaces marginaux, des situations dans lesquelles ont pu aboutir quelques espaces de liberté et de différence. L'objectif de cet article est de comprendre comment le rôle marginal du cirque a joué un rôle clé dans la popularisation du transformisme.

Le cas du Circo Timoteo représente le résultat d'un syncrétisme culturel, d'héritage européen et mésoaméricain, autour de l'emploi de l'humour et des rôles sexuels, dans une approche beaucoup plus complexe que celle d'une réalité sociale simplifiée et univoque. La figure du transformiste acquiert dans le cirque chilien un espace propre, par rapport à l'ambigüité des rôles sexuels et sociaux, depuis un syncrétisme culturel propre. La différence gagne sa place depuis le regard de l'indéfinition de la marge.

En premier lieu, nous allons comprendre théoriquement, le rôle ambigu du transformisme dans le cirque au Chili, depuis sa stigmatisation jusqu'au pouvoir obtenu par la satire. Deuxièmement, nous allons nous centrer sur l'analyse historique et ethnographique du cas du Circo Timoteo, depuis la dualité entre la considération du transformiste comme une particularité du cirque chilien ou son inclusion comme maladie contre les bonnes mœurs. Troisièmement, nous pointerons l'analyse linguistique des discours des transformistes du Circo Timoteo, pour démontrer l'ambivalence complexe des discours, dans la construction d'un espace de différence particulier.

Mots clé: cirque; différence; place; transformisme; métissage.

\section{Abstract. The place for difference in Chile: Circus and cross-dressing. The case of the Timoteo Circus}

The Chilean social model has traditionally left little room for formal differences. In certain marginal spaces, however, there are instances in which some niches of freedom and tolerance have been able to flourish. This article aims to understand how the marginal role of the circus was instrumental in popularizing cross-gender roles.

The Timoteo Circus is the result of a cultural syncretism between the European and Mesoamerican heritage around humor and sexual roles and is much more complex than a simple and unambiguous formal social reality. In this Chilean circus, the cross-gender figure adopts its own space based on the ambiguity of sexual and social roles through cultural syncretism. Difference occupies a place thanks to the margin of formal ambiguity. 
We first attempt to theoretically examine the ambiguous role of cross-gender in the Chilean circus from its stigmatization of gender to its empowerment through mockery. Secondly, we explore the ambivalence of the Timoteo Circus from both a historical and ethnographical viewpoint as both a genuine element of the Creole Chilean circus and as a social disease. Thirdly, we analyze the discourses of the Timoteo Circus cross-dressers to demonstrate their complex ambivalence as a way to build a specific space for difference.

Keywords: circus; difference; place; cross-dressing; syncretism.

\section{Sumario}

Introducción al rol del transformista en el circo criollo chileno

La paradoja del Circo Timoteo
Conclusión. El margen de la ambigüedad como lugar para la diferencia

Bibliografía

Discursos sobre la diferencia en Chile

\section{Introducción al rol del transformista en el circo criollo chileno}

El lugar de la diferencia en el contexto chileno ha sido escaso y marginal, pero ha encontrado un curioso refugio en el mundo del circo $^{1}$. El caso del transformismo en el circo, en el denominado "subgénero del circo show», es un excelente ejemplo para demostrar el espacio carismático y popular que adquiere el transformista en una sociedad tradicionalmente formal. Esta ambivalencia se produce frente a la complejidad de los discursos y se ha denominado usualmente «doble estándar» chileno. El circo permite la legitimación del transformismo desde su espacio marginal de libertad respecto del modelo tradicional formal.

El artículo se presenta como un ensayo analítico-interpretativo cuyo objetivo principal es intentar entender los motivos de la aparición y el rol que desempeñan los transformistas en el circo show chileno, a partir del caso emblemático del Circo Timoteo. En un contexto formal tradicional como el chileno, cuesta entender el gran apoyo popular de este tipo de espectáculos que incluye los públicos de las edades más variadas, desde niños hasta adultos mayores, tanto hombres como mujeres, así como de ámbitos tanto rurales como de barrio. La popularidad especial del Circo Timoteo lo ha convertido en un referente a escala nacional y ello ha dado pie a una proliferación de circos más chicos que se han especializado en este subgénero o han incluido transformistas en algunos

1. El artículo es un resumen de una investigación más amplia donde se intentan explicar las causas del surgimiento del género del circo show en Chile, así como el resultado de un proceso cultural criollo de mestizaje de valores europeos y mesoamericanos entorno al humor y la sexualidad (Prats, 2006). Para poder indagar en esa idea, la investigación se elaboró desde un trabajo etnográfico más amplio, así como con la ayuda del análisis lingüístico de algunos diálogos de espectáculos (varios DVD) y textos vinculados con el caso de estudio. Para acortar la extensión, se excluyó la historia del circo en Chile, la de la evolución del transformismo y la del punto de encuentro entre ambas, que se centra en la bohemia porteña de Valparaíso desde el siglo xix. 
de sus espectáculos. El Circo Timoteo se caracteriza por implementar una serie de rutinas, establecidas íntegramente con espectáculos de transformistas, que van desde números musicales hasta parodias. Además, la larga tradición de este tipo de espectáculos, junto con su gran receptividad, llevó a realizar una investigación de carácter exploratorio para indagar sobre los motivos profundos de su existencia y su razón de ser, lo cual condujo a adentrarse a estudiar, por un lado, la evolución tanto del mundo del circo, como del transformismo en Chile, y, por otro lado, analizar los factores de su éxito, a partir de un análisis profundo de los roles sociales y sexuales desde una perspectiva más culturalista.

En este sentido, el artículo no se planteó como un fin en sí, sino que abre el debate sobre una serie de temáticas poco abordadas en el campo de la geografía y aún menos en el contexto latinoamericano en general y el chileno en particular. La perspectiva del transformismo, a pesar de sus implicancias con la geografía del género y, en particular, con los queer-studies, ha sido considerada en este caso, desde una comprensión culturalista, un reflejo de ciertas formas de sincretismo cultural. No obstante, el tema del transformismo en el caso presentado también abre un interesante debate entorno a una aproximación no anglosajona al tema de los roles sexuales, desde una perspectiva de la teoría de la comunicación basada en las dialécticas de la ambigüedad o la complejidad de los discursos.

En consecuencia, el presente artículo debe ser entendido como un ensayo introductorio que analiza e interpreta dicho caso desde una perspectiva cultural (Clua y Zusman, 2002) y un abordaje de la teoría de la comunicación como campo de estudio en sí (Craig, 1999). Esta perspectiva compleja replantea seguramente muchos de los conceptos establecidos en algunas áreas de estudios culturales (Luna, 1999), de género (Butler, 1990) y aún más desde la geografía (Garcia Ramon, 2006), pero abre nuevos horizontes para un reflexión crítica (Soja, 2000).

En definitiva, el primer objetivo del artículo es intentar explicar la curiosa interrelación que se forjará en Chile entre el transformismo y el circo. Se hará a partir de una documentación bibliográfica sobre la historia del género en común y del transformismo en particular, así como sobre el mundo del circo en el caso chileno, que nos conduce a la cuna porteña del Valparaíso del siglo XIx. En segundo lugar, nos centramos en el estudio del caso específico del Circo Timoteo, a partir de la realización de un trabajo etnográfico que implicó la asistencia a algunas de las representaciones, entrevistas con algunos de los implicados, así como recopilación de material vinculado al caso de estudio, tales como prensa o páginas web. Finalmente, para entender el rol del discurso complejo ambivalente o «doble estándar» como elemento clave para la explicación del intersticio de margen de libertad, en el espacio del circo, como refugio para los transformistas, se analizaron los discursos lingüísticos a partir de extractos de diálogos de los espectáculos, procedentes de actuaciones en directo, así como de varios DVD editados o materiales difundidos directamente en la red, tales como vídeos. El tercer elemento de análisis es el que nos permite cruzar el caso de estudio concreto del Circo Timoteo con las dialécticas más teóricas, 
respecto, por un lado, del rol del discurso complejo en relación con los roles sexuales (más vinculado con los estudios de género), así como, por otro lado, respecto de la búsqueda de elementos del sincretismo cultural que refleja el transformista en el circo desde una perspectiva más culturalista.

Antes que nada, tenemos que recordar que el circo se ha establecido en su historia como el espacio de refugio de las diferencias ${ }^{2}$. Su situación ambulante respecto a modelos sedentarios lo ha asociado con una noción de espacio periférico o al margen, en el cual pudieron encontrar amparo todos aquellos que no tenían cabida en el modelo establecido. Dentro de ese contexto cultural e histórico en Occidente, el presente artículo presenta el caso particular de un circo compuesto de espectáculos de variedad, realizado íntegramente por travestis y enfocado a todos los públicos, como es el caso del Circo Timoteo, en Chile. El escenario del circo se convertirá en el espacio en el cual la estigmatización del género cruzado podrá adquirir, con su visibilidad social, no sólo un lugar, sino también un púlpito donde poder satirizar los códigos de la formalidad de los roles y las convenciones sociales establecidos. El circo y el transformismo en su indefinición adquieren, así, un lugar al margen de la norma.

\section{La estigmatización del género cruzado}

En los estudios de género y, más recientemente, en los queer studies, se ha puesto de manifiesto la incipiente complejidad de las interrelaciones posibles entre identidad y función de género (Browning, 1998). Dentro de esos márgenes posibles de masculinidad y feminidad (Arango et al., 1995), se establece igualmente un amplio espectro que se ha denominado "género cruzado" y que incluiría conceptos amplios de transformismo (cross-dresser), travestismo y todas las formas posibles de transexualidad (Brown y Rounsley, 1996). En el caso de estudio, se utiliza genéricamente el concepto travesti (travestido masculino) para aludir al concepto específico de transformismo. La diferencia principal entre ambos conceptos radica en que el transformismo acentúa más

2. En el mundo occidental, el carácter itinerante del circo ha llevado, por lo general, hacia una marginación de este universo respecto del modelo general sedentario establecido (Jacobs, 2002). El caso latinoamericano repite el tipo europeo, aunque generará algunas curiosas adaptaciones divergentes del modelo original, debidas al fruto de la mezcla de substratos culturales diversos. En el contexto latinoamericano, han ido surgiendo nuevas derivaciones del circo europeo. Así, por ejemplo, en el caso argentino, el circo surgirá desde la tradición circense italiana y francesa llegada a mediados del siglo XIX y generará un circo propio denominado "criollo" (Franco, 1982), que incluirá una serie de personajes característicos en la versión urbana bonaerense y en la gaucha en el interior del país (Castagnino, 1953). De la misma manera, podríamos hablar, en el contexto chileno, de una tradición de circo criollo propio que difiere de la tradición argentina y cuyas influencias no sólo remontarían a la tradición europea, sino igualmente a la inclusión de un abordaje del humor criollo, fruto del sustrato occidental y mesoamericano. En el contexto chileno, el circo mantiene su carácter popular, tanto en el contexto urbano como en las áreas rurales, aunque la mayor cantera de compañías se ha concentrado tradicionalmente en la quinta región, en estrecha vinculación con Valparaíso. 
la faceta de actuación y disfraz frente al otro (como función), mientras que el travestismo, se plantea como una forma de vida (como identidad). Ese matiz, como veremos en el caso de los personajes travestidos de los espectáculos del circo, se da de forma compleja, de modo que puede haber transformistas y travestidos, y todo ello independientemente de la conducta sexual, heterosexual u homosexual, aunque usualmente se identifique popularmente con la segunda (Gressgård, 2010). El margen de ambigüedad respecto a la sexualidad y a los roles se establece sobre unos discursos totalmente distintos de los parámetros de una identidad de género reivindicativa del modelo anglosajón, sobre el cual se ha establecido buena parte de los queer studies.

El circo de travestidos en Chile, o autodenominado "circo show», representa una adaptación y una interrelación de los marcos conceptuales del humor con el género, lo cual forja una forma criolla de circo específicamente chilena. El circo utiliza el género para poder replantear, en clave humorística, todos los roles sociales e identitarios fijados, y lo hace con una parodia social, desde su margen. Para poder entender la construcción de esa interrelación, debemos plantear la ambivalencia de los conceptos de humor y género que se han construido a partir de substratos culturales variados, lo que lo diferencia del modelo unitario occidental europeo.

Respecto al humor, el personaje del pícaro (Welsford, 1935), recogido en la tradición literaria española, fusiona con los personajes igualmente embaucadores (trickster) de las tradiciones indígenas (Durwin, 2004), como los que se encuentran en los cuentos del Ngürü (zorro) mapuche, entre otros. En el segundo caso, el engaño deriva en parte del disfraz y de la indefinición de género de muchos de los personajes, sobre los cuales sacan ventaja (Steward, 1931). Esa diferencia es entendida como una ventaja respecto al resto (Parsons y Beal, 1934). Desde esa perspectiva, la ambigüedad de forma y de género, común en numerosas culturas originarias de Mesoamérica, replantea la consideración del género como un juego de roles (Kirby, 1974) que son tomados de forma positiva e incluso, en algunos casos, mágica (Charles, 1945). De todo ello, podemos concluir que, por un lado, humor y género se entrelazan y, por otro lado, que ciertas formas de género cruzado se entienden como rol o bien como un prodigio divino (Hansen, 2001) (figura 1).

En el ámbito americano, la imposición paulatina del modelo occidental europeo, influenciado por la definición unidireccional de las identidades y las funciones de género cristianas, se va a sobreponer sobre los discursos previos más complejos, pero dejando márgenes para formas ambivalentes. En el mundo occidental, la identidad de género se reduce a su función biológica y reproductiva, y considera pecado cualquier otra vía, mientras que, en los modelos de Mesoamérica, el género se va a entender como roles, en un marco más amplio y con connotaciones espirituales y mágicas (Durwin, 2001). La irrupción del modelo occidental en América llevará a una estigmatización de las formas anteriores, entendidas como salvajes e inmorales, aunque terminará, en muchos casos, con la aceptación tácita de formas ambivalentes en la denominada «doble moral». Así, mientras se impone un 


\begin{tabular}{|c|c|c|c|}
\hline \multicolumn{2}{|c|}{ HUMOR } & \multicolumn{2}{|c|}{ GÉNERO } \\
\hline Occidental & Nativo & Occidental & Nativo \\
\hline Pícaro & Ngürü & $\begin{array}{c}\text { Biología } \\
\text { Reproducir } \\
\text { Pecado }\end{array}$ & $\begin{array}{c}\text { Rol } \\
\text { Placer } \\
\text { Magia }\end{array}$ \\
\hline \multicolumn{4}{|c|}{ CRIOLLO } \\
\hline \multicolumn{2}{|c|}{ Humor } & \multicolumn{2}{|c|}{ Género } \\
\hline \multicolumn{2}{|c|}{$\begin{array}{l}\text { Pedro Urdemales } \\
\text { (pícaro, Tricker) }\end{array}$} & \multicolumn{2}{|c|}{$\begin{array}{c}\text { Huebeo } \\
\text { (firteo ambiguo, calentura) }\end{array}$} \\
\hline \multicolumn{4}{|c|}{ Circo criollo } \\
\hline
\end{tabular}

Figura 1. La configuración criolla del humor y del género en Chile. Fuente: elaboración propia a partir de Arango et al., 1995.

orden estricto social en las formas, se tolerarán, de puertas hacia adentro, las otras conductas.

De todas esas mezclas culturales en temas de humor y de género, surgirán nuevas formas criollas propias que podemos vincular, por un lado, con el uso del humor burlesco. Estas formas han permanecido en personajes populares como Pedro Urdemales ${ }^{3}$, aún presente en el folclore chileno - desaparecido de la tradición originaria aragonesa- y en el uso de un lenguaje vivo y sagaz, lleno de picardías, dobles sentidos y alusiones sexuales, que podemos encontrar reflejado desde los pasquines ${ }^{4}$ hasta las payas ${ }^{5}$ o el lenguaje común popular.

3. Pedro Urdemales, peyuco,

Venido de chiloé,

Se encontró con una vieja

Que bailaba trucu trucutrucu

Y al dar la vuelta el peyuco,

Se retira más atrás,

La viejita lo miraba

Con el trucu trucutrucu.

Anda pues Pedro Urdemales

Con tus mentiras al diablo

Y vete por la sombrita

Que por el sol trucu trucutrucu.

«Pedro Urdemales» — cueca chilota (folklore chileno) -

en El folklore y la pasión, de Violeta Parra (EMI 830971-2).

4. Panfleto satírico.

5. Arte poético musical espontáneo, trova. 
Por otro lado, la identidad criolla de género se vuelve más ambigua, a pesar de conservar unos códigos sociales estrictos. Los roles de género son muy rígidos en su forma, tanto como en la sociedad colonial, y se agudizan incluso posteriormente respecto a Europa. Ello a pesar de que, por debajo, se esconda una laxitud tanto en el abordaje del género, como en las licencias, desde un modelo machista que abusará de la fuerte estratificación social como forma de imposición y dominio, el denominado coloquialmente «doble estándar». En el caso de Chile, se recogen testimonios de abusos de patrones sobre sus empleadas chinas, de ciertas licencias homosexuales de la élite santiaguina sobre poblaciones marineras como en la caleta de El Horcón o de los desmanes en la bohemia porteña, al margen de las normas de la buena moral impuesta en el siglo XIX y con fuertes influencias del modelo británico victoriano, que no hacen más que reflejar una posición de dominio de fondo y de forma de las clases mejor situadas sobre el resto.

La fusión de estas formas criollas de humor y género derivan hacia un sistema complejo de interacciones que difieren de los modelos europeos de origen y que nos permiten contextualizar el caso de estudio del circo show. Podemos encontrar, en elementos chilenos como la figura de artesanía del indio-picaro, esa misma mezcla entre lo burlesco y lo grotesco, basculando desde la comicidad hasta una forma de discriminación o exclusión. Esas formas criollas reflejan un sincretismo difícilmente comprensible desde los patrones europeos y de los pueblos nativos de origen, por lo cual representan formas y códigos sociales nuevos respecto al humor y al género (Willeford, 1969).

Lo burlesco adopta, en el circo show, el travestismo como soldado de batalla. La posición de ambigüedad de género, o mejor de intercambio de género, permite confundir los roles y tergiversar los códigos sociales y sexuales hasta reducirlos al absurdo. El travestismo es abordado desde las contradicciones propias de los discursos complejos de unas sociedades criollas que resultan de procesos sincréticos, sobre referentes y códigos culturales diversos. En ese contexto, el subgénero burlesco del travestismo en Chile puede ser entendido tanto como una forma de discriminación y estigmatización, como, a su vez, una forma de sociabilización y familiaridad entorno al género cruzado. Esto se refleja, por ejemplo, en el hecho que los payasos (Towsen, 1976) y los personajes transformistas del circo show adoptan roles sexuales invertidos para, por un lado, caricaturizar los estigmas discriminatorios hacia todas las formas de género cruzado y, por otro lado, a la vez, representar una visibilidad y un acercamiento popular hacia la diversidad (figura 2).

La inversión de rol sexual de los artistas del circo show les brinda una neutralidad de género que les ampara para abordar todos los tabús formales sobre los roles de género. Esa curiosa situación, lejos de ser novedosa, retoma algunas tradiciones de pueblos nativos, que encuentran, en el género cruzado o el disfraz y la ambigüedad, la forma de intermediación con otras dimensiones espirituales, con lo cual se convierten en los mediadores entre vivos y muertos. Así testimonian relatos sobre el carácter de género cruzado masculino de la mayoría de los machi ('curandero'), aunque la ulterior incursión de la Iglesia y 


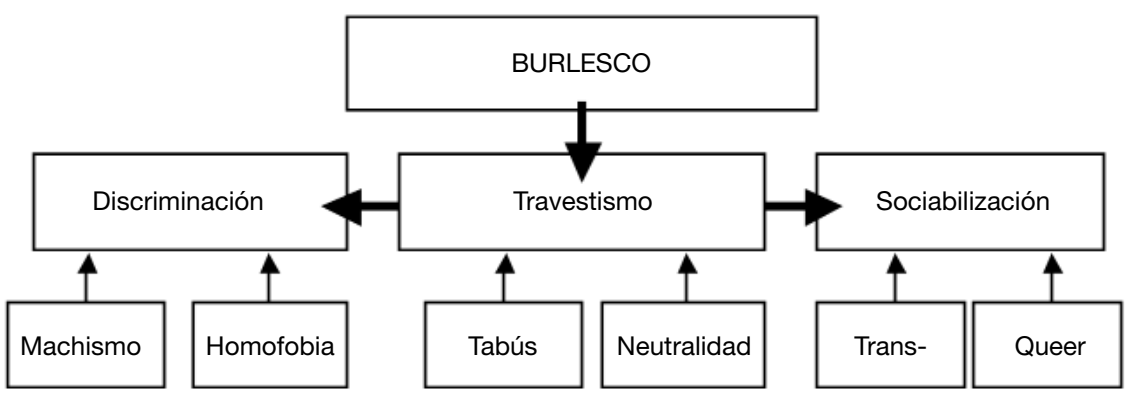

Figura 2. La relación de lo burlesco y el travestismo en la identidad criolla. Fuente: elaboración propia a partir de Durwin, 2001.

la imposición de la correlación entre función e identidad de género terminaría por atribuir el rol de machi a las mujeres (Bacigalupo, 2002).

En este sentido, los artistas del circo show representan un sincretismo de diversas tradiciones culturales. El artista transformista se convierte en un mediador en la parodia cómica no sólo de los estereotipos en contra de las formas de género cruzado, a partir de chistes discriminatorios hacia los homosexuales y los travestis, sino que igualmente reproduce los esquemas de un patrón machista sobre la mujer (Barberá, 1998). No obstante, esa forma de discriminación, al ser representada desde la inversión de roles, deriva hacia una burla de los mismos esquemas discriminatorios, puesto que aborda los tabúes de una sociedad machista que queda ridiculizada bajo el sesgo del humor (Barberá y Martínez-Benlloch, 1998).

La parodia del transformista termina convirtiéndose, entonces, en una sátira de los estándares de género, deja al descubierto la absurdidad del modelo y acerca al espectador a una complicidad hacia la diferencia, puesto que deja visibilizar el género cruzado y la homosexualidad. Tras los números de sátira, podrán alternar otros de vedettes transformistas que bailan y cantan sin causar ningún temor en los espectadores.

\section{La burla del transformista}

La paradoja del artista del circo show versa sobre cómo la transformación de la identidad sexual del referente en el discurso machista y estigmatizante respecto al género puede terminar ridiculizando los roles de la masculinidad y enfatizando los de la feminidad, la homosexualidad y el género cruzado. La neutralidad que aporta el circo como lugar especial y efímero, junto con la ambigüedad de género del artista, permite generar un distanciamiento espacial y catártico respecto de los códigos y de estándares sociales, detrás de las risas de los espectadores (Seibel, 2005). El transformista estigmatizado en cualquier esquina de la ciudad se convierte en personaje popular y querido, al ser capaz de encontrar un lugar donde mostrarse ante los demás y poder tener las agallas de decir en 
voz alta lo que muchos piensan en silencio sobre el género. La persecución y la estigmatización de las conductas de género desviadas del patrón establecido patrifocal (Garcés, 1936), al ser caricaturizadas por los mismos aludidos, lejos de reafirmar, terminan convirtiéndose en una sátira liberalizadora para los espectadores, así como para el actor, y obtiene un reconocimiento social tras la risa de su público. El circo ofrece una tarima para el reconocimiento y un lugar para la diferencia. El éxito de todo ese proceso pasa por conseguir la complicidad del público en general, pero particularmente la de las mujeres.

La caricaturización de la mujer por parte de los transformistas puede ser concebida como una reificación insultante de la femineidad desde la visión masculina, aunque curiosamente es en ellas donde el artista va a encontrar el mayor apoyo, al conseguir destronar, con su sátira, el modelo patriarcal que la mujer padece en su vivir diario (Ferreday, 2008). La comicidad en la confusión de roles de payasos varones ante los transformistas, la idealización de la mujer como icono femenino, así como la denuncia ácida de muchos de los abusos del machismo sobre las mujeres, despierta la risa femenina. Con el apoyo de ellas, los actores transformistas pueden avanzar de la misma forma en su espectáculo hacia la caricaturización del género cruzado, lo cual da cabida a una aceptación de los márgenes dentro de los códigos de género establecidos, con la inclusión, por un lado, de travestis, transexuales y, por otro lado, de formas de homosexualidad, sobre todo masculina.

\section{La paradoja del Circo Timoteo}

Dentro del circo show chileno, destaca el Circo Timoteo, tanto por su tradición, como por su popularidad. A pesar de su marginación formal en los nuevos estatutos del arte circense (2007), ese circo representa seguramente uno de los productos artísticos más propios del circo criollo (Castagnino, 1953) y, a la vez, con mayor trascendencia popular, tanto directa (público), como indirecta (a partir de las incursiones de algunos de los personajes de su circo en otros ámbitos del espectáculo, por ejemplo: en la televisión o en shows de locales gays).

El Circo Timoteo, a pesar de crear escuela, sigue lidiando entre la popularidad de ser un circo criollo de mucha aceptación y la persecución formal de un modelo de valores donde no cabe la ambigüedad de roles de género (Franco, 1982). A pesar de ello, el Circo Timoteo sigue adelante y constituye seguramente uno de los espectáculos más conocidos de los chilenos. Prueba de ello es la amplia difusión, en los estratos sociales más populares, de los varios DVD realizados (http://www.timoteo.cl/) sobre los espectáculos con los que genera más empatía. Incluso también se amplía a los estratos sociales que lo repudian en la forma, pero que lo compran para verlo en casa siguiendo un modelo del doble estándar. La clave del éxito radica en haber sabido sintetizar las características más particulares del pueblo chileno entorno al humor y el género (figura 3).

La transparencia cada vez mayor de los modelos de género cruzado en los medios de comunicación occidental en general, junto con todos los avances 




Figura 3. La popularidad del Circo Timoteo va más allá de su carpa. Fuente: vídeo del Circo Show Timoteo dedicado a Fabiola del Luján, editado por Oreval, Santiago.

en las reivindicaciones de los derechos hacia las minorías sexuales que se originaron en el mundo anglosajón a mediados de los años sesenta (disturbios de Stonewall de 1969), podrían llevarnos a pensar que el circo show en Chile es una consecuencia de ello. Pero este tipo de espectáculo, por el contrario, responde a una lógica particular de larga tradición que debemos hacer remontar a la bohemia porteña del Valparaíso del siglo xix y que seguramente tiene, incluso, precedentes anteriores.

\section{Luces: un elemento del circo criollo chileno}

Para entender el Circo Timoteo, nos tenemos que remontar al surgimiento de la Bohemia de Valparaíso en el siglo xIx. Escala casi obligada en la ruta transoceánica, el intercambio de mercancías fue el origen del auge del puerto de Valparaíso desde fines del siglo XVIII hasta fines del XIX. La ulterior apertura del canal de Panamá reformularía los flujos comerciales en detrimento de Chile. La pujanza económica anterior no sólo se manifestó en el crecimiento de la ciudad, sino también en su carácter cosmopolita. Cabe señalar que la llegada de inmigrantes y, en especial, las colectividades alemana e inglesa dejarían mucha huella en la ciudad. De igual forma, no se puede dejar de mencionar el trajín de marineros y comerciantes de toda población porteña, que, tras la travesía del Cabo de Hornos, querían encontrar entretenimiento. 


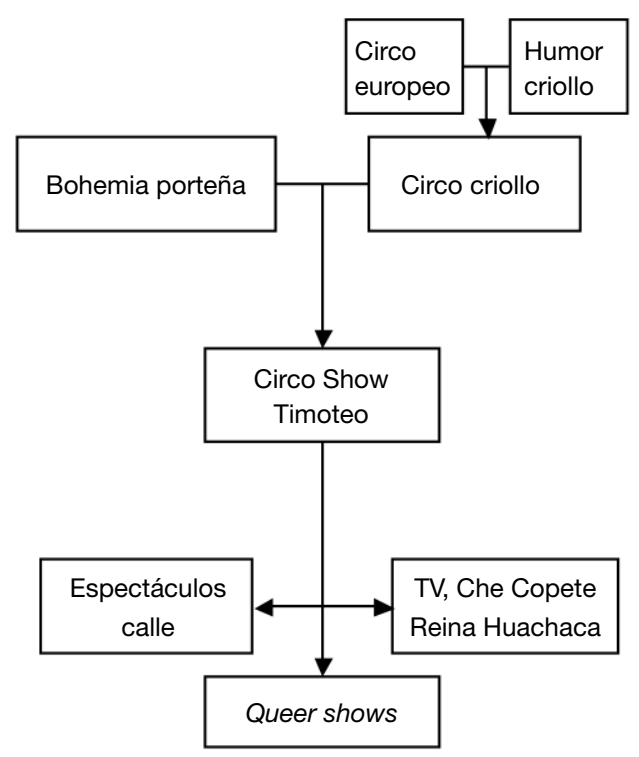

Figura 4. La génesis y evolución del circo show. Fuente: elaboración propia.

Todos estos elementos condujeron al auge de la vida cultural y de la entretención de Valparaíso, con la proliferación, entre otros, de hoteles, cafés, teatros, bares, tabernas, cantinas y hasta burdeles que, a pesar de las numerosas disposiciones para su control y erradicación, terminarían por dar la identidad y el mal renombre de ciertos barrios y locales de la ciudad porteña, como, por ejemplo: el Roland Bar, El Violeto, la Casa Amarilla, los 7 Espejos, el América Bar, el Flamingo Rosa, el Bar Chile o el Nunca se Supo, entre otros.

Esta confluencia terminó forjando una bohemia nocturna en Valparaíso que trascendería hasta el levantamiento militar de 1973, con personajes emblemáticos como Petra Pérez o el mismo Violeto (transformista), que daría nombre a su local. Dentro de ese contexto de diversión, proliferaron los cabarés y espectáculos varios, que permitieron dar un espacio a transformistas que representaban números de variedades.

La aparición del Circo Timoteo en 1968, a cargo de sus fundadores René Valdés (Timoteo) y Darío Zúniga, retomará parte de toda esa tradición del transformismo porteño que se mezclará igualmente con la tradición circense gestada de igual forma en la quinta región. El circo europeo llegado por el puerto de Valparaíso se irá adaptando a las pautas de un humor criollo fruto de un mestizaje de elementos heredados del tiempo colonial, así como tradiciones nativas de la zona de los changos (Castro, 2001). El circo criollo chileno permitirá que se difunda el transformismo hacia áreas rurales e interiores del país, lo cual propicia la articulación del género popular del circo show. El Circo 
Timoteo, en la actualidad, retoma esa tradición y se convierte en el modelo para otros circos más chicos que han desarrollado este género en la zona central de Chile, principalmente.

Por un lado, el impacto y la relevancia de ese subgénero del circo se ha difundido ulteriormente en los medios de comunicación (televisión), en personajes como Che Copete o en vodeviles y comedias ligeras, lo cual ha propiciado la aparición de una subcultura popular denominada "huachaca», fuertemente criticada por ciertos sectores de la cultura (Huneeus, 2008).

Por otro lado, la liberación progresiva de minorías sexuales (movimiento gays y trans) a partir del fin del Gobierno militar, en la década de 1990, propiciaría la inserción de otros personajes del Circo Timoteo (como, por ejemplo, Katiuschka Molotov) en el mundo del espectáculo, en discos y bares gays - en Valparaíso y Santiago principalmente-, así como la apertura de un nuevo espacio para el transformismo (shows de transformistas, de drag queens y, en menor medida, de drag kings), con lo cual se convirtió en bandera de una forma de visibilización de las reivindicaciones de los colectivos gays. Este proceso no está exento de polémica, puesto que el abordaje del tema gay se inserta dentro de una lógica de reivindicación de la diversidad, diferente del contexto ambivalente criollo.

El Circo Timoteo sintetiza una larga tradición criolla y, a la vez, ha sido una escuela para la difusión de todo un género de espectáculos en Chile que hoy se refleja desde algunos circo shows hasta programas televisivos, la venta de varios DVD, los espectáculos en discos gays o incluso la realización de un reality de transformistas por Internet. La difusión gracias a los medios de comunicación, junto con el cambio de tendencia respecto a la estigmatización del género cruzado y la homosexualidad desde los modelos occidentales, principalmente europeos, además de la derogación de leyes discriminadoras o la igualación de derechos para las minorías sexuales, ha permitido acrecentar su aceptación más allá de los sectores sociales más populares, incluyendo la clase media, aunque aún sigue estigmatizada por las élites y los círculos más ortodoxos de las iglesias (católicos y protestantes).

\section{Sombras: una enfermedad de trascendencia social}

Las élites no consideraron al circo como un arte equiparable a otras formas culturales, y su carácter marginal y diverso fue muchas veces estigmatizado. La evolución de las políticas de inserción del rubro circense dentro de las regulaciones laborales estandarizadas en Chile muestra justamente como, detrás de la legalización, sigue prevaleciendo una forma encubierta de discriminación desde esa élite legisladora. Así, la regularización de los estatutos del rubro obedece, por un lado, a la búsqueda de una mejora para la comunidad del circo, en un intento de paliar la precariedad asociada a su marginalización, aunque, por otro lado, igualmente, la estandarización encubre un discurso solapado de orden moral, en una imposición de un orden sobre cualquier forma de vida diversa, entendida como desviada del modelo establecido. Una cosa es mejorar 
las condiciones de vida del circo y otra distinta es imponer una forma de vida amparándose en la primera.

Una de las curiosidades es observar como, a pesar de los cambios políticos y de ideología de los gobiernos sucesivos, se sigue manteniendo el mismo tipo de discurso coercitivo detrás de la norma. Desde esa perspectiva, no sorprende encontrar, dentro de los estatutos del trabajador del circo de 1935, alusiones mucho más allá de la normativa, asociando tácitamente el espectáculo con las malas costumbres y las enfermedades de trascendencia social, en un fiel reflejo de la proyección de las ideas higienistas de la época al campo de lo social. Se intenta, así, justificar científicamente las formas morales de orden social establecidas o de buena conducta sobre el circo.

Para contextualizar esa visión, sirvan otras alusiones de la época para controlar los desmanes sociales en el discurso de higienismo social de Montengro ${ }^{6}$, más cercanos a los discursos europeos de fines del siglo XIX que a los del primer cuarto del siglo $\mathrm{xx}$.

A pesar del cambio de mentalidades y tendencias políticas actuales, no deja de sorprender como, en 2007, en un nuevo intento de regularizar el trabajo de los artistas del circo, se aprovechó para vetar todos los espectáculos circenses de denotado contenido adulto, con lo cual se intentaba terminar con el circo show. Setenta años después del primer estatuto ${ }^{7}$, sigue la búsqueda encubierta de la privación de las libertades de expresión y artísticas, bajo el pretexto de una mejora de las condiciones de los artistas del circo.

En el extracto de la ley aprobada en 2007, se observa como se sigue discriminando el género circense vinculándolo con la frivolidad, intentando, además, subdividir el circo show del resto del circo para generar una guerra interna. Aunque lejos de lo esperado, algunos de los principales circos tradicionales, como el Tachuela, a pesar de quedar bien amparados en el nuevo estatuto, salieron en defensa del circo show, y en particular del Circo Timoteo, como

6. «[...] Grandes esfuerzos de oratoria y nutridos artículos de prensa pretenden poner atajo a la ola de corrupción que amenaza acabar con las energías de la raza. Higienistas, moralistas y personas de buena voluntad, ven el peligro cierto de las enfermedades de carácter social, y convienen en que es llegado el momento de emprender una acción conjunta de todas las fuerzas vivas e inteligentes de la nación contra este "Hannibal ad portas" que amaga la vida física, moral y espiritual de la sociedad chilena. Pero todos, aún los más ilustrados y de mejores intenciones, se han dedicado a la cura de los "efectos", olvidándose extirpar previamente las "causas"..." (Montengro, 1922).

7. «[...] Para los efectos de la presente ley, se entiende por circo aquellos establecimientos preferentemente habilitados en carpas que, debidamente autorizados, están destinados a la celebración de espectáculos circenses y cuya programación se orienta especialmente a los niños. Se entenderá por espectáculo circense la ejecución o representación en público de ejercicios físicos de acrobacia o habilidad, de actuación de payasos, malabaristas, prestidigitadores e ilusionistas, músicos, animales amaestrados y otras similares.

En tal virtud, esta actividad podrá acceder, a través de los mecanismos legales pertinentes, a los recursos que contempla la legislación vigente en materia de fomento de expresiones artísticas y culturales. No tienen el carácter de circos los espectáculos de contenido frívolo o de revista, orientados al público adulto, aun cuando ellos sean presentados en carpas...» (Extracto de la Ley 20216). 
parte del circo chileno; aunque igual manifestaron un repudio hacia ciertos circos chicos que derivan a veces en otras actividades paralelas. En este proceso de dignificación del rubro, una parte de la diversidad refugiada en el circo en Chile ha visto peligrar su futuro, a pesar del enorme apoyo popular.

En conclusión, podemos comprobar como se sigue reflejando el repudio de las autoridades hacia la consideración formal de la diversidad de formas de vida. La inserción del circo en la sociedad sigue concibiéndose desde el abandono obligado de su diferencia, en vez de poder constituirse desde el propio reconocimiento formal. Paradójicamente, cuesta más el aval oficial que el aplauso general.

\section{Discursos sobre la diferencia en Chile}

El testimonio del circo show representa, dentro del espectro del circo chileno, un curioso dilema de difícil encaje dentro de los parámetros formales (Sussman, 1998). Para poder entender mejor el surgir de esta forma de diferencia en un contexto supuestamente tan adverso, se analizará el propio discurso de los circo shows desde el caso del Circo Timoteo. Los temas y el abordaje de las temáticas permitirán observar la ambivalencia del discurso de la diferencia en Chile, en una curiosa simbiosis entre una dialéctica burlesca y una actuación del transformista que desarma los discursos discriminadores al cambiar el interlocutor. La esencia del éxito de ese tipo de espectáculo yace en el haber sabido llegar a la esencia del humor criollo, al descontextualizar el discurso formal estigmatizante, manteniendo su apariencia, en un ágil guiño de engaño sagaz.

\section{La ambivalencia compleja de discursos}

Por diversos motivos, la ambivalencia de los diálogos en los espectáculos del circo show puede llegar a desconcertar a cualquier persona no acostumbrada a ellos. En primer lugar, el registro semántico y de léxico tiende a ser muy grosero. En segundo lugar, el contenido de los diálogos puede llegar a ser muy ambiguo. En tercer lugar, la puesta en escena y la participación de los espectadores permite fraguar los dos aspectos anteriores, lo cual genera el efecto burlesco deseado.

El lenguaje ágil y grosero, con alusiones explícitas a temas sexuales así como escatológicos, es el argumento sobre el cual muchas veces ciertos sectores de élite se han respaldado para marginar este tipo de espectáculo como adulto. No obstante, ese tipo de diálogo recoge no sólo una tradición surgida del lenguaje popular y de la calle, sino también de un humor criollo, de larga tradición en Chile y vinculado a una herencia de estirpe colonial y nativa. El lenguaje sagaz, trufado de dobles sentidos, con connotaciones sexuales y uso de palabras groseras, no sólo se presenta en este tipo de espectáculos en Chile, sino también, desde los pasquines del siglo xIx hasta las payas en la cueca chilena, sin descuidar el uso de un lenguaje y de expresiones muy picantes de algunas de las lenguas nativas, como el mapudungun o el quechua. En ese contexto, 
el uso de ese tipo de registro, si bien es vetado desde la visión occidental tradicional, debiera ser reconsiderado desde otras lógicas culturales, donde estas características se entienden como formas de interjección exagerada o hipérbole. Son formas para llamar la atención de un interlocutor, desde su picardía y comicidad, y que no deben ser tomadas nunca en sentido literal.

A continuación, transcribimos algunos fragmentos de un espectáculo del Circo Timoteo, para ejemplificar justamente estos registros de lenguaje y poder analizar más adelante la ambivalencia del contenido de los diálogos:

[...] Damas lindas mariconeando [...] soy enferma del poto pero no del hocico [...] yo sé que hoy aquí hay mucha gente que viene por la novedad... y hay muchos weones ${ }^{8}$ que vienen a reírse de los maricones [...] pero cuando llegan acá se les agacha el mojón porque pucha que es difícil este arte de nosotros que es transformar, arreglarnos para hacer reír al público [...] esas son las mujeres de Chile que quieren y aman a los maricones [mujer del público entrega espontáneamente un ramo de flores al artista] porque el mejor amigo de las mujeres es el mariconcito [...] aunque nunca los tengan muy cerca puesto que salen cagando con el marido [...] yo no porque me los como cagando en el váter $[\ldots]$

[...] Veo más mujeres que hombres, que chucha pasa acá... Me estoy dando cuenta que somos más mujeres esforzadas, dueñas de casa, cuidando marido y a tragalás de hombres que les cagan [...] yo menos mal que hice una manda. En unos 10 años no quiero tener hombres, tengo 2 perros doberman, ellos me lengüetean la raja [...] Ya me puse grosera [...] pero la chucha [al diablo] [...] en todo caso [...] la gente paga su entrada para venir a ver este show. Me pongo a observar y veo que mucha gente se pegan codazos y dicen: «Esa weona ${ }^{9}$ que tiene lindas piernas!», y la otra pregunta: «广Y tiene tetas?», y la otra más indiscreta: «¿Dónde tiene escondida la pichula?» [...]. Pero yo siempre cerca del pueblo [...]. Si a usted le gusta el pico, cómaselo [...] yo soy franca $[\ldots]$ hay las cartuchas ${ }^{10}$ y las que se hacen las leonas [...] aquí está la gente del pueblo. La gente pituca ${ }^{11}$ no la vemos. Son las que se tiran los pedos más hediondos. Yo me gusta mariconear. Yo me entrego tal como soy [...] Aquí veo mucha gente que le gusta esta clase de weveo [...] El «circo show» es para adultos $[\ldots]$ si vienen niños es responsabilidad de sus padres [...] aunque hoy en día, saben más los weones que uno [...] el niño pregunta: «¿Qué te cuelga? ¿la guata?». (Extracto de diálogo del vídeo de espectáculo del Circo Timoteo editado por Oreval.)

El contenido de los diálogos es seguramente uno de los aspectos más interesantes de analizar dentro de los espectáculos del circo show y, siguiendo una tradición criolla, demuestra el uso de la ambivalencia del lenguaje. En primer lugar, se observa como el lado ligero y rápido de los discursos tiene el propósito de parecer espontáneo, aunque se sustenta sobre una trama básica siguiendo
8. 'Giles'.
9. 'Pesada'.
10. 'Pudorosas'.
11. 'Acomodada'. 
las tradiciones teatrales más clásicas de la Commedia dell'Arte. La trama del diálogo y el personaje están establecidos, pero las florituras del lenguaje y la puesta en escena quedan sujetas a variaciones específicas en función del actor y del aforo.

Una de las primeras observaciones generales al analizar el diálogo es el hecho que, si no supiéramos que quien actúa es un transformista, pensaríamos que se trata de una burla machista y homofóbica. En ese sentido, los diálogos traducen fielmente los estereotipos estigmatizantes frente a la diferencia en la sociedad chilena, pero llevándolos a su exageración extrema. Los diálogos podrían incluso ser casi insultantes para los aludidos, por el tinte discriminatorio, pero la paradoja radica justamente en el hecho que el sujeto de todos esos diálogos es un actor transformista que, por su propia presencia y actuación, termina por desvirtuar y convertir en ridículos todos los estereotipos estigmatizantes. El hecho de parodiar la propia discriminación termina generando un efecto de catarsis que provoca la risa del público y, a la vez, también supone, en el caso de los actores transformistas, travestis y/o homosexuales, una forma de liberación frente a la marginación. El resultado de esos diálogos permite, a través del humor, conceder un lugar a la diferencia dentro de una sociedad clasista, estigmatizante.

El análisis del diálogo en su detalle desvela una interesante estrategia en el encadenamiento de las temáticas. En primer lugar, el actor va a ridiculizar algunos de los estereotipos machistas respecto del rol sumiso de la mujer. El transformista consigue la comprensión y la ulterior complicidad de las mujeres por ser igualmente dominadas, dentro del modelo tradicional, por causa de su género. De esta manera, las espectadoras pueden reírse de muchas de las expresiones y experiencias sufridas a diario frente a sus propios maridos, con lo cual el show se transforma en una liberación de los roles de género para la mujer.

A partir de ese momento, el actor lleva el diálogo hacia el tema más complejo de la homosexualidad y del transformismo. Para conseguir arrancar la risa del espectador, en ese caso, no sólo utilizará las mismas estrategias de burla de los diálogos clásicos estigmatizantes, sino que también va a involucrar a los propios espectadores varones. El actor jugará con la ventaja del miedo escénico del espectador para ridiculizarlo frente a todo el aforo al sentarse sobre sus rodillas o darle golpes con una cartera, por ejemplo. El símbolo del rol masculino queda totalmente desvirtuado frente al rol del transformista y se genera una inversión de los roles de poder en la representación de los géneros.

Finalmente, en la última parte de la transcripción del extracto anterior, se aborda el tema tabú de la sexualidad y los roles de género frente al público infantil. Recordemos que, además, desde 2007, los estatutos del circo han intentado mermar el circo show, arguyendo justamente el carácter adulto de sus espectáculos. En ese contexto, el actor, en un giro genial, desarma los miedos de la sexualidad ante los más chicos de una forma cómica y a la vez legal, al responsabilizar a cada adulto de la presencia de sus hijos. En esta ocasión, los temas sexuales son abordados desde la utilización de la ingenuidad infantil, como doble sentido, cuestionando el grado real de inocencia del menor. El 
análisis del diálogo del espectáculo permite evidenciar como, a partir del rico manejo de registros y actuación, se puede abordar la ambigüedad de roles, no sólo de género, sino también sociales.

Si bien algunos estudios han intentado tipificar la actividad del transformismo, vemos, en el caso de estudio del circo show, como las fronteras son mucho más ambiguas. Esa característica es justamente la particularidad del caso chileno. En vez de categorizar la diversidad en espacios segregados, como en el modelo anglosajón, por ejemplo, nos encontramos con un modelo homogéneo estigmatizante que ha dejado toda diferencia en su margen. Dentro de esa diferencia, situamos el lugar del género cruzado y, más en particular, el papel de los transformistas. El caso de Chile responde a una aproximación del transformismo compleja en cuanto a noción, identidad, contexto y motivaciones, respecto de otros modelos (figura 5).

Así, por ejemplo, el transformista en el Circo Timoteo es una simbiosis ambivalente de todas las categorías atribuidas a sus roles y funciones posibles: puesto que es un artista y/o puede llegar a expresar sus propias emociones y/o simboliza un homenaje a la mujer y/o legitima al travestismo y/o a las formas de género cruzado. El artista transformado (cross-dresser) representa un rol con independencia de su condición sexual, sea heterosexual u homosexual, y de su función de género en la sociedad fuera del espectáculo, sea travestido o transexual. Ese abanico de posibilidades e interrelaciones resume la complejidad de situaciones y a la vez representa un resquicio de tolerancia, casi paradójico, dentro del contexto discriminador chileno. El circo show es un espacio al margen del modelo unitario y representa una paradoja, incluso para los modelos sociales tolerantes de otros países, donde la diversidad es muchas veces tipificada y encapsulada en discursos unívocos, como en la interrelación de travestismo con homosexualidad o prostitución.

En el contexto chileno, el travesti puede encontrar, curiosamente, un abanico de opciones múltiples y de reconocimiento público y social, según como, mayor que en contextos más liberales, a pesar del reducido ámbito de tolerancia chileno. Las formas de sexo cruzado no ven sus opciones laborales de vida reducidas a un ámbito de shows en discotecas de ambiente gay, o simplemente en el rubro de la prostitución, sino que han encontrado un refugio controversial en el ámbito circense. La ambigüedad y la complejidad de situaciones dentro del transformismo del circo chileno le permiten justamente obtener una relevancia y una visibilidad social mucho mayor que en contextos sociales más tolerantes. Si bien en este contexto no podemos hablar de aceptación, sí que, en cambio, podemos ver que, en el modelo chileno, el reconocimiento del rol es mayor, aunque nunca el de su función.

El caso del circo show representa una adaptación de una tolerancia en los roles desde un abordaje unívoco del género de raíz cristiano-occidental. Esa ambivalencia de discursos y roles es una característica que podría considerarse diferente a la criolla y que refleja el substrato pluricultural de Chile y de Latinoamérica, en sentido amplio. La ambigüedad se establece a partir del desdoblamiento de roles por parte de un mismo sujeto, lo cual genera, por un lado, la 


\section{Categorías relacionadas directamente a la actividad del transformismo.}

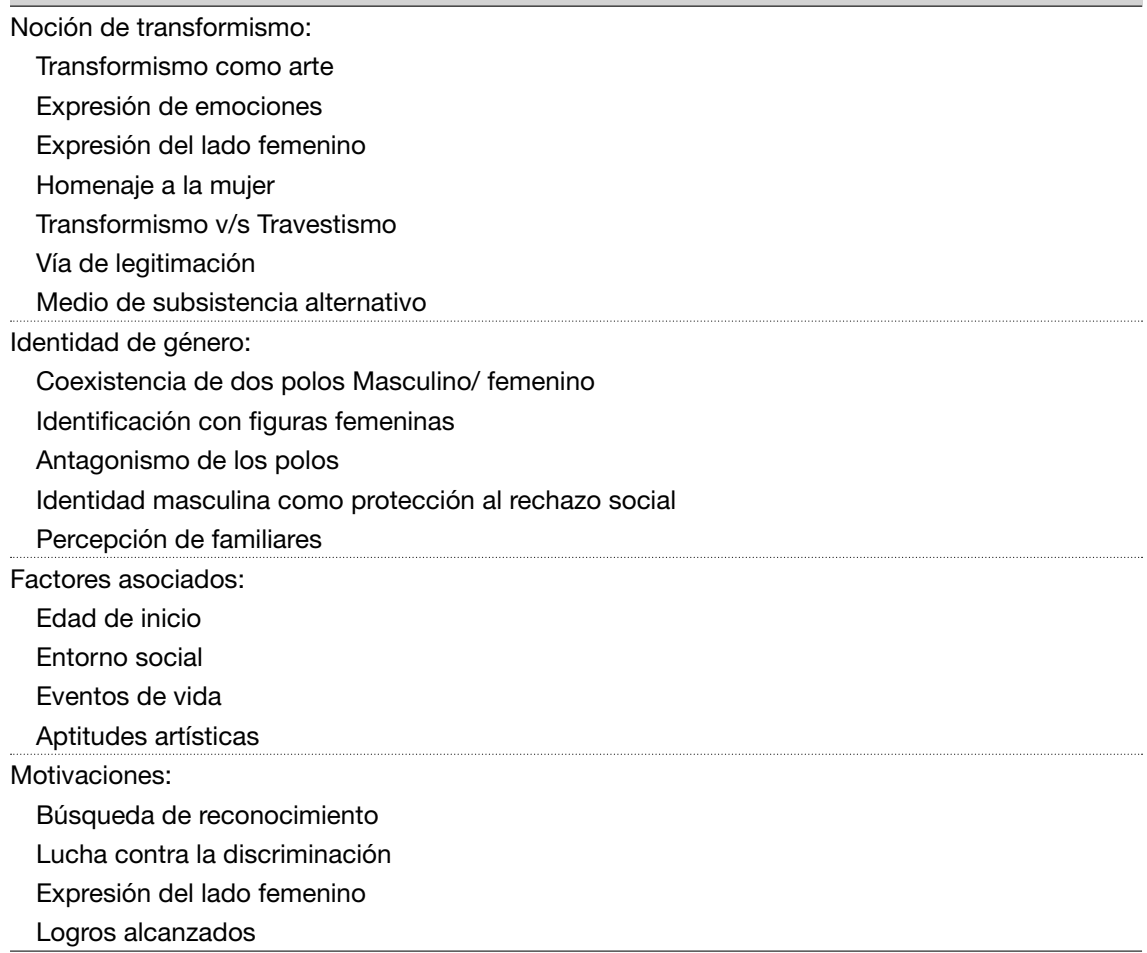

Figura 5. La diversidad criolla: ambigua y compleja. Fuente: Álvarez, 2009.

multiplicidad de papeles y funciones de género que se pueden establecer y, por otro lado, por su solapamiento, bajo un modelo formal machista inquebrantable. Esto conduce a una libertad de facto para el varón en sus roles y funciones, así como a una dependencia unívoca para los demás, léase mujeres y géneros cruzados. El caso del circo show refleja un espacio para el transformismo con márgenes para discursos intermedios, lejos de los modelos unívocos, dualistas y excluyentes de género, de raíz occidental y cristiano-occidental, que podrían pensarse a priori en el contexto chileno.

\section{La diferencia a la criolla}

El circo, a pesar de su marginación, es una de las grandes suertes para la sociedad chilena y ha representado una burbuja de libertad dentro de un modelo casi asfixiante respecto a las conductas sociales y morales. El lugar del circo representa casi una tercera vía alternativa a los dualismos de una sociedad marcada por el maniqueísmo, heredado del modelo colonial y que se expresa en 
binomios como hombre-mujer, patrón-capataz, bueno-malo, y que se adaptó desde modelos culturales mesoamericanos igualmente binarios, aunque con mayor matiz.

El circo se convirtió en el refugio de formas de vida diversas y, en lo concreto, dio sustento a un grupo de personas estigmatizadas por su vivencia diversa del género. La libertad del margen de opinión albergó en el circo a un colectivo formalmente proscrito y definió un espacio de acercamiento de otras visiones. Además, su popularidad se extiende no sólo en los barrios urbanos populares, sino también en el medio rural, factores que subrayan su raigambre y tradición.

El circo show evidencia una forma indirecta de reconocimiento de formas de género cruzado, en una sociedad que formalmente repudia terceras vías. Esa es la respuesta a la criolla del acercamiento a la diferencia, basada en la ambivalencia de discursos múltiples desde un mismo sujeto varón. La complejidad de los discursos convive con la claridad de un modelo unívoco, lo cual genera un margen de ambigüedad que permite un acercamiento para todos, aunque deba ceñirse a determinados códigos (lugares y tiempos definidos). Fuera de esos intervalos, la estigmatización persiste aunque en el margen, el humor aporta una versatilidad y una informalidad del discurso que permite una flexibilización social y de género, al igual que actúan los carnavales para Brasil o Bolivia, por ejemplo.

Los transformistas son una realidad, pero en el circo se convierten en una necesidad para, por un lado, amortiguar, así como, por otro lado, perpetuar la rigidez de un modelo formal unívoco de sociedad. Sirva, entonces, este artículo para denotar la importancia del circo show ante las autoridades, al igual que cualquier otra forma de expresión cultural, al margen de los discursos formales.

\section{Conclusión. El margen de la ambigüedad como lugar para la diferencia}

En el presente artículo, se ha indagado en la comprensión de la paradoja de la existencia del Circo Timoteo en un contexto muy marcado por conductas sociales rígidas, desde una perspectiva culturalista que nos ha llevado a indagar en el curioso sincretismo cultural criollo entorno a los códigos vinculados con el humor y con los roles sexuales.

En el presente caso de estudio, la indefinición del circo respecto a las pautas sociales convencionales se convirtió en la arena que no sólo dio refugio al travestismo, sino igualmente una forma de sociabilización y popularidad. La ambigüedad de los discursos del transformismo desde este lugar al margen del modelo establecido, aporta, desde la sátira, la parodia necesaria sobre el modelo de códigos sociales rígidos establecidos. El circo y el transformista se convierten, curiosamente, en una necesidad para poder garantizar la permanencia del modelo social formal. Desde el intersticio espacial del circo, desde la ambigüedad de los roles sexuales del travestismo, así como también desde la ambigüedad del discurso de la sátira criolla, se construye un rico lugar de diferencia que ocupará un espacio central en las confinadas vidas diarias de muchos chilenos. 
Esta investigación inductiva, desde una argumentación diálogo-dialéctica, permite, más allá del caso de estudio concreto, abrir una puerta a la reflexión para otros estudios en temáticas poco abordadas, desde perspectivas académicas transversales y con una reconsideración hacia el planteamiento idiográfico para la comprensión del mundo contemporáneo (Baudrillard, 1986), al margen de ideas preestablecidas.

\section{Bibliografía}

Álvarez, Nancy y Pérez, Carmen (2009). «Identidad de género en transformistas: un estudio cualitativo-exploratorio». Limite, 4 (20), 123-152.

Arango, Luz; León, Magdalena y Viveros, Mara (1995). Género e identidad: Ensayos sobre lo masculino y lo femenino. Colombia: Uniandes.

Bacigalupo, Ana Mariella (2002). «La lucha por la masculinidad del machi: Políticas coloniales de género, sexualidad y poder en el Sur de Chile». Revista de Historia Indigena, 5, 29-64.

BARBerá, Ester (1998). Estereotipos de género: Construcciones de las imágenes de las mujeres y los varones. Madrid: Pirámide.

Barberá, Ester y Martínez-Benlloch, Isabel (1998). Psicología del género. Madrid: Ariel.

Baudrillard, Jean (1986). América. Barcelona: Anagrama.

Brown, Mildred y Rounsley, Chloe Ann (1996). «True Selves: Understanding Transexualism for Families, Friends, Coworkers and Helping Professionals». En: The Transsexual Dilemma. San Francisco: Jossey-Bass.

Browning, Frank (1998). «Do Gays Exist?». En: A Queer Geography: JourneysToward a Sexual Self. Nueva York: The Noonday Press, Farrar, Straus and Giroux.

ButLer, Judith (1990). Gender trouble: Feminism and the subversion of identity. Nueva York / Londres: Routledge.

Castagnino, Raúl Hector (1953). El circo criollo: Datos y documentos para su historia 1757-1924. Buenos Aires: Lajouane.

Castro, V. (2001). «Atacama en el tiempo: Territorios, identidades, lenguas». Anales de la Universidad de Chile, VI (13), 27-70.

Charles, Lucille (1945). «The Clown's Function». Journal of American Folklore, 58 (227), 25-34.

Clua, Anna y Zusman, Perla (2002). «Más que palabras: otros mundos. Por una geografía cultural crítica». Boletín de AGE, 34, 105-117.

Craig, Robert T. (1999). "Communication theory as a field». Communication Theory, 9 (2), 199-161.

Durwin, Joseph (2001). Dreamtime: Psychobiological Methodology and Morphogenesis in the Shamanic Tradition. Berkshire County Community College. The Orbits Project.

- (2004). «Coulrophobia and the Trickster». Trickster's Way. San Antonio: Trinity University, 3 (1), 1-22.

Ferreday, Debra (2008). «Showing the girl: The new burlesque». Feminist Theory, 9 (1), 47-65.

Franco, Lily (1982). El circo criollo. Buenos Aires: Mágicas Ruinas.

GarcÉs, Francisco (1936). Estatutos del sindicato circense. Santiago de Chile: La Gratitud Nacional. 
Garcia Ramon, Maria Dolors (2006). «Geografía del género». En: Hiernaux, Daniel y Lindón, Alicia. Tratado de Geografía Humana. Barcelona: Anthropos, 337-355.

GressGÅRD, Randi (2010). "When trans translates into tolerance - or was it monstrous?: Transsexual and transgender identity in liberal humanist discourse». Sexualities, 13, 539-561. Sociology Department at the University of Essex.

Hansen, George (2001). The Trickster and the Supernatura. Xlibris Corporation.

Huneeus, Pablo (2008). Cultura Huachaca o el aporte de la televisión. Santiago: Nueva Generación.

Jacobs, Pascal (2002). La Fabuleuse Histoire du Cirque. París: Hachette.

Kirby, E.T. (1974). "The Shamanistic Origins of Popular Entertainments». The Drama Review, 18 (1), 5-15.

Luna García, Antonio (1999). «¿Qué hay de nuevo en la nueva geografía cultural?». Documents d'Anàlisi Geogràfica, 34, 69-80.

Montengro, M.J. (1922). "La enfermedad de trascendencia». Claridad. Universidad de Chile, 2 (65).

Parsons, Elsie y Beal, Ralph (1934). "The Sacred Clowns of the Pueblo and MayoYaqui Indians». American Anthropologist, 36 (4), 491-513.

Prats, Maria (2006). «Sexo, género y lugar». En: Nogué, Joan y Romero, Joan (eds.). Las otras geografias. València: Tirant Lo Blanch, 511-526.

Seibel, Beatriz (2005). Historia del circo. Buenos Aires: Ediciones del Sol.

SojA, Edward (2000). Postmetropolis: critical studies of cities and regions. Los Angeles: Blackwell.

Steward, Julian (1931). «The Ceremonial Buffon of the American Indian». Papers of the Michigan Academy of Science, Arts, and Letters.

Sussman, Mark y Cohen-Cruz, Jan (dir.) (1998). Queer circus: Amok in New York: Radical street performance: an international anthology. Nueva York: Routledge, 262-269.

Towsen, John (1976). Clowns. Prentice-Hall.

Welsford, Enid (1935). The Fool: His Social and Literary History. Londres: Faber and Faber.

Willeford, William (1969). The Fool and His Scepter: A Study in Clowns and Jesters and Their Audience. Northwestern University Press.

\section{Bibliografia web}

Última consulta: 15 de diciembre de 2010

http://circo.cl/?page_id=1101

http://www.timoteo.cl/

http://www.opusgay.cl/1315/printer-72465.html

http://www.glbtq.com/social-sciences/geography.html 